\title{
Gas Kinetic Scheme for Anisotropic Savage-Hutter Model
}

\author{
Wen-Chi Chen ${ }^{1}$, Chih-Yu Kuo ${ }^{1, *}$, Keh-Ming Shyue ${ }^{2}$, Yih-Chin Tai ${ }^{3}$ \\ ${ }^{1}$ Division of Mechanics, Research Center for Applied Sciences, Academia Sinica, Taipei 115, Taiwan \\ ${ }^{2}$ Department of Mathematics, National Taiwan University, Taipei 106, Taiwan \\ ${ }^{3}$ Department of Hydraulic and Ocean Engineering, National Cheng Kung University, Tainan 701, \\ Taiwan
}

\begin{abstract}
The gas-kinetic scheme is applied to a depth-integrated continuum model for avalanche flows, namely the Savage-Hutter model. In this method, the continuum fluxes are calculated based on the pseudo particle motions which are relaxed from non-equilibrium to equilibrium states. The processes are described by the Bhatnagar-Gross-Krook (BGK) equation. The benefit of this scheme is its capability to resolve shock discontinuities sharply and to handle the vacuum state without special treatments. Because the Savage-Hutter equation bears an anisotropic stress on the tangential space of the topography, the equilibrium distribution function of the microscopic particles are shown to be bi-Maxwellian. These anisotropic stresses are shown the key to preserve the coordinate objectivity in the Savage-Hutter model. The effect of the anisotropic stress is illustrated by two examples: an axisymmetric dam break and a finite mass sliding on an inclined planary chute. It is found that the propagation of the flow fronts significantly depends on the orientation of the principal axes of the tangential stresses.
\end{abstract}

Keywords: Gas-kinetic scheme, BGK equation, extended Savage-Hutter equation

\section{Introduction}

Landslides, avalanches, and debris flows are gravity-driven rapid geophysical flows. Because these flows commonly exhibit the characteristics of shallowness, the shallow-water type of equations are often applied to model the phenomena. In addition, the flows contain a large portion of solid particles and present solidlike behavior. To account for the solid effects, Savage \& Hutter [1] propose to use the Mohr-Coulomb soil constitutive law for the landslide or avalanche materials in the shallow-water continuum model. When the flow are in motion, the basal friction causes the materials to move at the yield condition on the basal surface. With the internal friction angle and the Mohr-Coulomb yield criterion, the stresses in the tangential plane to the basal surface are calculated and incorporated into the momentum equation.

The Savage-Hutter model is subsequently extended for two-dimensional channel topography, Hutter et al. [2], Pudasaini \& Hutter [3], Wang et al. [4]. The model equations form a conservative hyperbolic system. Because this type of partial differential system can contain discontinuous weak solutions, e.g. shock waves (hydraulic jumps), numerical scheme needs to be able to resolve these weak solutions accurately. There are several common categories of solvers, for example, the high-order (approximate) Riemann solvers, LeVeque [5], Toro [6], and the non-oscillatory central finite differences schemes, Jiang

Email: ${ }^{1}$ olivech@gate.sinica.edu.tw (W.C. Chen),

Email: ${ }^{1, *}$ cykuo06@gate.sinica.edu.tw (C.Y.. Kuo),

Email: ${ }^{2}$ shyue@math.ntu.edu.tw (K.M. Shyue),

Email: ${ }^{3}$ yctai@mail.ncku.edu.tw (Y.C. Tai). 
et al. [7], Tai et al. [8]. In addition, particularly for landslide flow applications, wet-dry states are often encountered such as in the landslide flows of a finite mass reported in the previous literature.

In this paper, we aim to extend the kinetic scheme, $\mathrm{Xu}$ [9], to the Savage-Hutter model. In this method, the flow fluxes across the interfaces between computational cells are simulated by the motions of microscopic pseudo particles. These pseudo particles move along with their microscopic velocities and are subjected to perfectly elastic collisions. Under such circumstances, the density distribution function of the particles is assumed to follow the approximate Boltzmann equation, the Bhatnagar-Gross-Krook equation. Taking statistical moments of these pseudo particle motions yields the mass and momentum fluxes in the continuum regime. The benefit of this scheme is its capability to resolve shock discontinuities sharply, the positiveness of the flow depth, and to handle the wet-dry state without the need of special treatments.

With the Mohr-Coulomb soil constitutive law and through the theoretical derivation of the depth averaged model $[2,10,11,12]$, the stresses in the tangential plane to the basal surface are related to the flow depth and basal friction. The principal axes of the stresses are in general dependent on the local flow conditions, i.e. the tangential stresses are anisotropic. To simplify the complexity of this solid property for numerical simulations, Hutter et al. [2], Tai et al. [8], as well as Wang et al. [4] align the primary principal axis along the flow channel direction and the minor axes in the transverse direction. Though the approach simplifies the numerical scheme, the model becomes coordinate-dependent, as commented in Hutter, Wang and Pudasaini [13]. To amend this deficit, a variety of models have been proposed.

There are three main categories of these amending theories: Iverson and Denlinger [14] model the tangential stresses of an fluid element as isotropic active (passive) soil stresses if the element is in dilation (compaction). De Toni and Scotton [15], and Kelfoun and Druitt [16] assume that the primary principal axis of the tangential stresses is in parallel to the local flow velocity. Luca et al. [12] propose that the principal axes coincide with the those of the local strain rate. In the latter two variants, the anisotropic properties of the tangential stresses are retained.

In applying the gas-kinetic scheme, we can investigate the effects of the anisotropic stresses as well. This leads to the bi-Maxwellian distribution of the pseudo particles. Such kind of anisotropy has been seen in the plasma and astrophysics where the multi-temperature state can be realized. What may differ from the situation considered here physically is that, in the plasma- and astrophysics, the particle motion is restrained by the electric-magnetic fields, resulting in the temperature anisotropy.

The Savage-Hutter model is briefed in Sec. 2. The gas kinetic scheme are presented in Sec. 3, where the relation between the microscopic pseudo particle motion and the continuum anisotropy Savage-Hutter equation is established. In Sec. 4, the numerical scheme for isotropic inviscid fluid is verified and the coordinate-dependent property of the Savage-Hutter model, [2, 10, 11, 8], is demonstrated. Subsequently in Secs. 4.3 and 4.4, we investigate the effect of the direction of the stress principal axes by an axisymmetric dam break flow and apply De Toni's amendament for the flow of a finite mass sliding down an inclined flat chute. It is shown the anisotropic stresses influences the flow spreading significantly.

\section{Savage-Hutter model}

The mass and momentum balance equations of the Savage-Hutter model for shallow, cohesionless granular flows (cf. $[1,13,17])$ can be written, respectively, as

$$
\begin{aligned}
\frac{\partial h}{\partial t}+\operatorname{div}(h \mathbf{u}) & =0 \\
\frac{\partial}{\partial t}(h \mathbf{u})+\operatorname{div}\left(h \mathbf{u} \otimes \mathbf{u}+\frac{g_{3} h^{2}}{2} \mathbf{K}\right) & =h \mathbf{S},
\end{aligned}
$$


where $h$ and $\mathbf{u}$ denote the depth and velocity of the avalanche flow. The reference coordinates $\mathrm{X}=\left(x_{1}, x_{2}\right)$ are idealy constructed to incline at the average slope of the basal surface. In this reference coordinates, the basal surface is described by $b=b(\mathrm{X})$ in the $x_{3}$-direction, which is defined normal to the $\mathrm{X}$-plane and, also along this direction, The flow depth is measured. The $x_{3}$-component of the gravitational acceleration in the flow direction is $g_{3}$ and the other component of the gravitational acceleration is $\mathbf{g}=g_{i} \mathbf{e}_{i}$ in the $\mathbf{X}$-plane. Symbol $\mathbf{K}$ denotes the matrix of the earth pressure coefficients which result from the Mohr-Coulomb soil constitutive law.

The source term $\mathbf{S}$ on the right-hand side of (1) takes the form

$$
\mathbf{S}=g_{i} \mathbf{e}_{i}-g_{3} \operatorname{grad} b-\mu g_{3} \frac{\mathbf{u}}{|\mathbf{u}|},
$$

where the first term is the gravitational driving acceleration and the second is the driving force due to the basal surface gradient. The last term is the resistive Coulomb friction force acting in the opposite direction of the flow. The basal friction coefficient is $\mu$ which is conventionally related to the basal friction angle $\delta$ by $\tan \delta$.

The general form of the earth pressure coefficients $\mathbf{K}$ is a $2 \times 2$ matrix

$$
\mathbf{K}=\left(\begin{array}{ll}
k_{11} & k_{12} \\
k_{21} & k_{22}
\end{array}\right)
$$

with entries $k_{i j}$ for $i, j=1,2$. This specific form can be obtained rigorously by the formal depth integration procedures presented in [12]. When applied in chute flows, Hutter et al. [2] and Wieland et al. [11] choose $x_{1}$ to coincide with the main flow direction and neglect the tangential earth pressure components by setting $k_{12}=k_{21}=0$. This simplification is equivalently to assume that the primary principal axis of the tangentail stresses is in the flow direction and the minor principal axis is in the cross flow direction $x_{2}$. Under such circumstances, $k_{11}$ and $k_{22}$ become the primary and secondary earth pressure coefficients, and the earth pressure coefficient matrix becomes

$$
\Lambda_{\mathbf{K}}=\left(\begin{array}{cc}
k_{1} & 0 \\
0 & k_{2}
\end{array}\right) .
$$

Using the Mohr-Coulomb soil yield condition, these two pressure coefficients are functions of the internal and basal friction angles, which can be written explicitly

$$
k_{1}=2 \sec ^{2} \phi \sqrt{1 \mp\left(1-\cos ^{2} \phi / \cos ^{2} \delta\right)}-1
$$

and

$$
k_{2}=\frac{1}{2}\left(k_{1}+1 \mp \sqrt{\left(k_{1}-1\right)^{2}+4 \tan ^{2} \delta}\right),
$$

where $\phi$ is the internal friction angle of the flow material. The minus-plus signs in the expressions are chosen according to the signs of $\partial u_{i} / \partial x_{i},(i=1,2)$, i.e. the streching or compaction of the flow element, [18]. For simplicity in the present paper, the active earth pressure coefficients are chosen (minus signs are taken).

When the previous assumption of the alignment of the principal axes is relaxed, the principal axes can in general align at any angle $\theta$ that the flow prefers. I.e. angle $\theta$ depends on the local flow conditions and varies somewhat according to the different models for the anisotropic stresses, [15], [16] or [12]. We neglect the microscopic gyro motions of soil particles in the present paper and this simplification leads to the symmetric earth pressure coefficient matrix, i.e. $k_{12}=k_{21}$. Therefore, $\mathbf{K}$ is diagonalizable by rotating the coordinates by $\theta$ with an invertible matrix $\mathbf{T}=\mathbf{T}(\theta)$ such that we have $\boldsymbol{\Lambda}_{\mathbf{K}}=\mathbf{T}^{-1} \mathbf{K} \mathbf{T}$. 
For conveniencing the following discussion, equation (1) is recast into the customary hyperbolic form

$$
\frac{\partial \mathbf{W}}{\partial t}+\frac{\partial \mathbf{F}_{1}}{\partial x_{1}}+\frac{\partial \mathbf{F}_{2}}{\partial x_{2}}=\mathbf{\Psi}
$$

where the column vectors $\mathbf{W}, \mathbf{F}_{1}, \mathbf{F}_{2}$ and $\boldsymbol{\Psi}$ are

$$
\begin{aligned}
\mathbf{W} & =\left(h, h u_{1}, h u_{2}\right)^{T}, \\
\mathbf{F}_{i} & =\left(h u_{i}, h u_{1} u_{i}+\frac{1}{2} g_{3} k_{1 i} h^{2}, h u_{2} u_{i}+\frac{1}{2} g_{3} k_{2 i} h^{2}\right)^{T}, \\
\mathbf{\Psi} & =\left(0, g_{1} h-g_{3} h \frac{\partial b}{\partial x_{1}}-\mu g_{3} h \frac{u_{1}}{|\mathbf{u}|}, g_{2} h-g_{3} h \frac{\partial b}{\partial x_{2}}-\mu g_{3} h \frac{u_{2}}{|\mathbf{u}|}\right)^{T},
\end{aligned}
$$

for $i=1,2$.

\section{Gas Kinetic scheme}

The gas kinetic scheme is constructed based on using statistical behavior of the motions of microscopic pseudo particles to mimick the conservation law of the continuum governing equation. The motions of the pseudo particles are described by the Bhatnagar-Gross-Krook (BGK) equation [19].

At the discretized level, the mass flux and momentum across the interfaces between adjacent computational cells are in general discontinuous and, as the time step marches, these physical quantities are transported into the cells according to the conservation law. In the scheme, this process is described by the redistribution process of pseudo microscopic particle motions from an non-equilibrium (initial) state into an equilibrium state. This is in a similar way as the redistribution process in the statistical mechanics but in contrast to the method based on the wave propagation, e.g., the Riemann solvers.

Prendergast \& Xu $[20,21]$ demonstrate the applicability of this type of the gas kinetic theory for compressible gas dynamics simulations and, subsequently, Xu [9] extends to the hydraulic shallow-water (SW) equations. In these approaches, the equilibrium distribution of the pseudo particles is assumed to be Maxwellian and the redistribution process is described by the BGK equation

$$
\frac{\partial f}{\partial t}+\mathbf{c} \cdot \operatorname{grad} f=\frac{g-f}{\tau},
$$

where $f$ and $g$ are the non-equilibrium and the equilibrium distribution functions, respectively. The relaxation time between the two states is denoted by $\tau$ and $\mathbf{c}=\left(c_{1}, c_{2}\right)^{T}$ is the velocity of the pseudo microscopic particles. In the present scheme, the external gravitational force is not balanced in the microscopic scale as in [9], but is treated in the continuum level, i.e. in the Godunov scheme.

Because of the anisotropic tangential stresses of the Savage-Hutter model, the pseudo particle motions are spatially asymmetric. For a two-dimensional domain, the equilibrium distribution of the pseudo particles at any given position is a bi-Maxwellian distribution with its principal axes coinciding with those of the Savage-Hutter equation. With this assumption, the equilibrium distribution can be written explicitly as

$$
g=\frac{1}{\pi g_{3} \sqrt{k_{1} k_{2}}} \exp \left\{-\frac{1}{g_{3} h}\left(\mathbf{c}^{\prime}-\mathbf{u}^{\prime}\right)^{T} \Lambda_{\mathbf{K}}^{-1}\left(\mathbf{c}^{\prime}-\mathbf{u}^{\prime}\right)\right\}
$$

where $\mathbf{c}^{\prime}$ and $\mathbf{v}^{\prime}$ are the micro- and macro-scale velocities along the principal axes and $\Lambda_{\mathbf{K}}$ is defined in (4). In the $\mathbf{X}$-coordinates, the distribution reads

$$
g=\frac{1}{\pi g_{3} \sqrt{k_{1} k_{2}}} \exp \left\{-\frac{1}{g_{3} h}(\mathbf{c}-\mathbf{u})^{T} \mathbf{K}^{-1}(\mathbf{c}-\mathbf{u})\right\}
$$


where $\mathbf{c}$ and $\mathbf{u}$ are the micro- and macro-velocities in the $\mathbf{X}$-coordinates and the coordinate transform $\mathbf{c}=$ $\mathbf{T}^{-1} \mathbf{c}^{\prime}$ and $\mathbf{u}=\mathbf{T}^{-1} \mathbf{u}^{\prime}$ is applied. This bi-Maxwellian distribution function can be theoretically obtained by assuming the pseudo particles are distributed at the maximum entropy condition, as recapitulated in Appendix A, and it is commonly seen in the plasma physics, usually resulting from the external restraining force fields, e.g. Xu et al. [22].

The bi-Maxwellian distribution function (10) is in general a function of the flow depth, $h$, velocity, $\mathbf{u}$, and the earth pressure coefficient matrix, $\mathbf{K}$, i.e. based on the conservative variable $\mathbf{W}=\mathbf{W}(\mathbf{X}, t)$. In addition to be expressed on a function of the flow variable, the distribution function can also be writen as a function of $(\mathbf{X}, t)$. We will choose whichever expression is appropriate in the following derivation. With this distribution, the continuum source-free (homogeneous) Savage-Hutter equations (1) can be reconstructed by taking the conservative moment integrals on the BGK equation at the limiting state $(f=g)$, see Appendix A. This property ensures that the gas kinetic scheme can be designed.

The gas kinetic scheme starts by solving for the non-equilibrium distribution function of the BGK equation (8). For a given relaxation time $\tau$, the distribution function reads

$$
f(\mathbf{X}, t)=f_{0}\left(\mathbf{X}_{0}, 0\right) \exp \left(-\frac{t}{\tau}\right)+\frac{1}{\tau} \int_{0}^{t} g(\hat{\mathbf{X}}, s) e^{\frac{s-t}{\tau}} d s,
$$

where the first term is the transient homogeneous solution to fulfill the initial distribution $f_{0}$ at $t=0$ and the second term is the particular solution in the convolution form with the equilibrium distribution g. Notation $\hat{\mathbf{X}}$ is defined as $\hat{\mathbf{X}}=\mathbf{X}-(t-s) \mathbf{c}=\mathbf{X}_{0}+s \mathbf{c}$, which is understood as the particle trajectory.

The continuum physical quantities can be constructed by taking the moment integral with respect to the column vector $\boldsymbol{\Theta}=\left(1, c_{1}, c_{2}\right)^{T}$ on the BGK equation which reads

$$
\int \boldsymbol{\Theta}\left(\frac{\partial f}{\partial t}+\mathbf{c} \cdot \operatorname{grad} f\right) d \mathbf{c}=\int \boldsymbol{\Theta} \frac{g-f}{\tau} d \mathbf{c}
$$

When at the equilbrium limiting state, $f=g$, the above integrals yield the homogeneous equations of the Savage-Hutter model as pointed out previously.

We now proceed the gas kinetic scheme. This scheme is used to solve for the homogeneous evolution of the Savage-Hutter equation. Its relation to the homogeneous Savage-Hutter equation is somewhat analogous to the zeroth order Chapmann-Enskog expansion of the idea gas BGK equation to the Euler equation. The scheme is designed to have a vanishing right hand side of (12) at all time, i.e. to satisfy

$$
\int \Theta \frac{(g-f)}{\tau} d \mathbf{c}=0
$$

This condition is referred to as the compatibility condition in literature [21].

In the numerical scheme, the computation domain is discretized into a set of uniformly distributed grids. For clarity, take a row of grids in the $x_{1}$-direction $(x)$ for illustration, see Fig. 1 . As shown in the figure, the subscripts $i$ and ${ }_{i+1}$ indicate the centers of the $i^{\text {th }}$ and $(i+1)^{\text {th }}$ cells, and the subscript $i+1 / 2$ represents the interface between the two cells. At the beginning of each time step, say $t=0$, the continuum flow variable in the two cells are denoted as $\mathbf{W}_{i}$ and $\mathbf{W}_{i+1}$ and, with a second order slope reconstruction, there are also $\partial \mathbf{W}_{i} / \partial x$ and $\partial \mathbf{W}_{i+1} / \partial x$ in each cell. Using these values, we approximate the initial non-equilibrium distribution function $f_{0}$ in the left and right cells as

$$
f_{0}(x, 0)=\left\{\begin{array}{cc}
g_{0}^{l}+\frac{\partial g_{0}^{l}}{\partial x}\left(x-x_{i+1 / 2}\right), & x \leq x_{i+1 / 2} \\
g_{0}^{r}+\frac{\partial g_{0}^{r}}{\partial x}\left(x-x_{i+1 / 2}\right), & x>x_{i+1 / 2} .
\end{array}\right.
$$




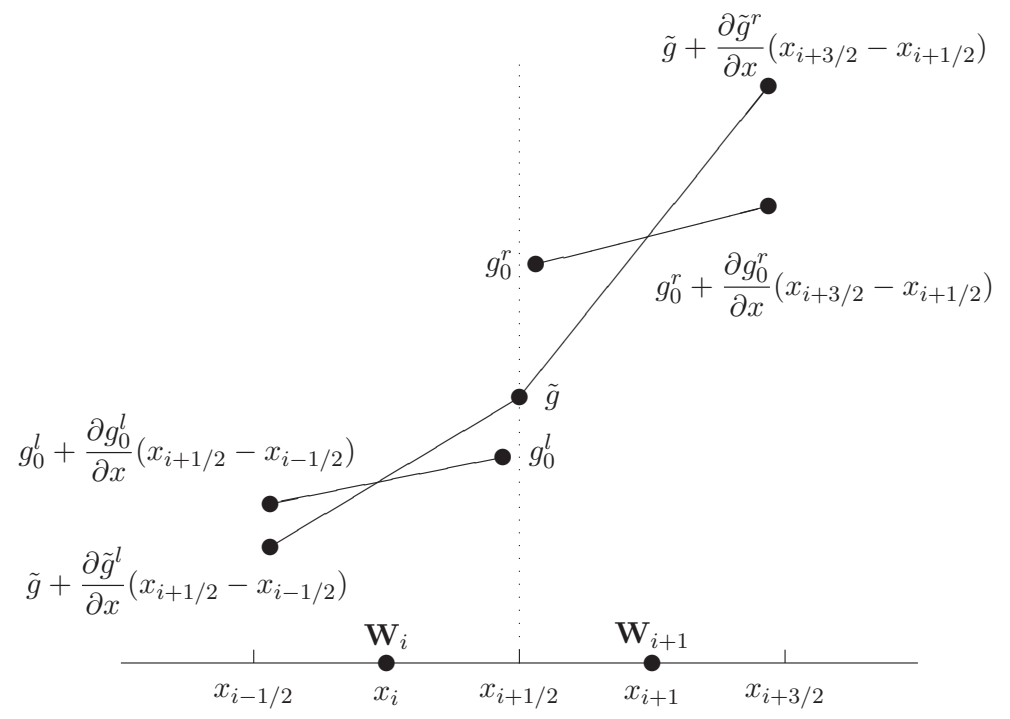

Figure 1: Initial discretized local non-equilibrium $f_{0}$ and equilibrium $g$ distribution functions at interface $x_{i+1 / 2}$.

This non-equilibrium state is achieved by the numerical update from the previous time step. The superscripts ${ }^{l}$ and ${ }^{r}$ represent that the distribution function is evaluated at the immediate left and right of the interface. I.e., $g_{0}^{l}$ and $g_{0}^{r}$ are $g_{0}^{l}=g\left(\mathbf{W}_{i}\left(x_{i+1 / 2}\right)\right)$ and $g_{0}^{r}=g\left(\mathbf{W}_{i+1}\left(x_{i+1 / 2}\right)\right)$ where $g$ is given by (10). Their spatial derivatives $\left(\partial g_{0}^{l, r} / \partial x\right)$ can be accordingly found with the aid of the continuum variables, $\mathbf{W}_{i}, \mathbf{W}_{i+1}, \partial \mathbf{W}_{i} / \partial x$, and $\partial \mathbf{W}_{i+1} / \partial x$. Without digression, the explicit expression of $\partial g / \partial x$ is given later, cf. (24).

On the other hand, there is an equilibrium distribution $g$ which is yet an unknown. It is assumed to be continuous across the interface and to satisfy the compatibility condition at each time step. The equilibrium distribution, hence, in the space-time neighborhood of the cells can be approximated to the second order as

$$
g(x, t)= \begin{cases}\tilde{g}+\frac{\partial \tilde{g}^{l}}{\partial x}\left(x-x_{i+1 / 2}\right)+\frac{\partial \tilde{g}}{\partial t} t, & x \leq x_{i+1 / 2}, \\ \tilde{g}+\frac{\partial \tilde{g}^{r}}{\partial x}\left(x-x_{i+1 / 2}\right)+\frac{\partial \tilde{g}}{\partial t} t, & x>x_{i+1 / 2} .\end{cases}
$$

In the above expression, $\tilde{g}$ is understood by the initial equilibrium state on the interface $x=x_{i+1 / 2}$ and is to be found by the compatibility condition (13) on the interface.

We are now to find each term on the right hand side of (15). Assuming that the relaxation time is independent of the particle velocity, (13) becomes

$$
\int \Theta \tilde{g} d \mathbf{c}=\int \Theta f_{0} d \mathbf{c}
$$

(16) on the interface at the initial time, after substitution of (14) and (15). The left hand side of (16) is in fact $\tilde{\mathbf{W}}_{i+1 / 2}$ on the interface and the right hand side integral is then calculated by the moments that the particles carry cross the interface. With $(14),(16)$ is readily recast into

$$
\tilde{\mathbf{W}}_{i+1 / 2}=\int_{c_{1} \geq 0} \Theta g_{0}^{l} d \mathbf{c}+\int_{c_{1}<0} \Theta g_{0}^{r} d \mathbf{c}
$$

Having obtained $\tilde{\mathbf{W}}_{i+1 / 2}$, the initial equilibrium distribution $\tilde{g}$ is solved to be $g=g\left(\tilde{\mathbf{W}}_{i+1 / 2}\right)$. Using $\tilde{\mathbf{W}}_{i+1 / 2}$ with $\mathbf{W}_{i}\left(x_{i}\right)$ and $\mathbf{W}_{i+1}\left(x_{i+1}\right)$, we find the slopes of the flow variables in the two cells $\partial \tilde{\mathbf{W}}_{i} / \partial x$ 
and $\partial \tilde{\mathbf{W}}_{i+1} / \partial x$ and subsequently the slopes, $\partial \tilde{g}^{l, r} / \partial x$, are defined, also with the help of (24). Finally, for $\partial \tilde{g} / \partial t$, we need the time derivative of $\tilde{\mathbf{W}}_{i+1 / 2}$ and it can be found by integrating (13) with (11), (14), and (15) on $x=x_{i+1 / 2}$. After some lengthy but routine calculation, $\partial \tilde{\mathbf{W}}_{i+1 / 2} / \partial t \mathrm{reads}$

$$
\begin{aligned}
\frac{\partial \tilde{\mathbf{W}}_{i+1 / 2}}{\partial t}= & \frac{\alpha \Gamma_{1}}{\Gamma_{0}}\left(\int_{c_{1} \geq 0} c_{1} \Theta \frac{\partial \tilde{g}^{l}}{\partial x} d \mathbf{c}+\int_{c_{1}<0} c_{1} \Theta \frac{\partial \tilde{g}^{r}}{\partial x} d \mathbf{c}\right)+ \\
& \frac{\alpha \Gamma_{2}}{\Gamma_{0}}\left(\int_{c_{1} \geq 0} c_{1} \Theta \frac{\partial g_{0}^{l}}{\partial x} d \mathbf{c}+\int_{c_{1}<0} c_{1} \Theta \frac{\partial g_{0}^{r}}{\partial x} d \mathbf{c}\right)+ \\
& \frac{\alpha \Gamma_{3}}{\Gamma_{0}}\left(\int_{c_{1} \geq 0} \boldsymbol{\Theta}\left(g_{0}^{l}-\tilde{g}\right) d \mathbf{c}+\int_{c_{1}<0} \boldsymbol{\Theta}\left(g_{0}^{r}-\tilde{g}\right) d \mathbf{c}\right),
\end{aligned}
$$

where the multiplying constants are

$$
\begin{aligned}
\Gamma_{0} & =\Delta t-\tau\left(1-e^{-\Delta t / \tau}\right), \\
\Gamma_{1} & =-\Delta t+2 \tau\left(1-e^{-\Delta t / \tau}\right)-\Delta t e^{-\Delta t / \tau}, \\
\Gamma_{2} & =\Delta t e^{-\Delta t / \tau}-\tau\left(1-e^{-\Delta t / \tau}\right) \\
\Gamma_{3} & =1-e^{-\Delta t / \tau} \\
\alpha & =k_{1} k_{2} /\left(k_{11} k_{22}+k_{12}^{2}\right) .
\end{aligned}
$$

and $\Delta t$ is the upper bound of the time integral. Noting that the anisotropic earth pressure coefficients lead to the extra coefficient $\alpha$. Again by referring to (24), with $\partial / \partial x$ replaced by $\partial / \partial t$, we obtain $\partial \tilde{g} / \partial t$.

The numerical fluxes across the interface $x_{i+1 / 2}$ can be obtained by taking moments of $f\left(x_{i+1 / 2}, t\right)$,

$$
\begin{aligned}
\mathbf{F}_{1(i+1 / 2)=} & \int c_{1} \boldsymbol{\Theta} f\left(x_{i+1 / 2}, t\right) d \mathbf{c} \\
= & \gamma_{0}\left(\int_{c_{1} \geq 0} c_{1} \Theta g_{0}^{l} d \mathbf{c}+\int_{c_{1}<0} c_{1} \Theta g_{0}^{r} d \mathbf{c}\right)+ \\
& \gamma_{1}\left(\int_{c_{1} \geq 0} c_{1}^{2} \Theta \frac{\partial g_{0}^{l}}{\partial x} d \mathbf{c}+\int_{c_{1}<0} c_{1}^{2} \boldsymbol{\Theta} \frac{\partial g_{0}^{r}}{\partial x} d \mathbf{c}\right)+ \\
& \gamma_{2}\left(\int_{c_{1} \geq 0} c_{1} \Theta \tilde{g} d \mathbf{c}+\int_{c_{1}<0} c_{1} \Theta \tilde{g} d \mathbf{c}\right)+ \\
& \gamma_{3}\left(\int_{c_{1} \geq 0} c_{1} \Theta \frac{\partial \tilde{g}^{l}}{\partial x} d \mathbf{c}+\int_{c_{1}<0} c_{1} \Theta \frac{\partial \tilde{g}^{r}}{\partial x} d \mathbf{c}\right)+ \\
& \gamma_{4}\left(\int_{c_{1} \geq 0} c_{1}^{2} \Theta \frac{\partial \tilde{g}^{l}}{\partial x} d \mathbf{c}+\int_{c_{1}<0} c_{1}^{2} \Theta \frac{\partial \tilde{g}^{r}}{\partial x} d \mathbf{c}\right),
\end{aligned}
$$

where $f\left(x_{i+1 / 2}, t\right)$ is the one-dimensional form of (11) and substitution of (14) and (15) has been made. As a reminder, the same procedure has also been applied in finding (18). The multiplying constants, $\gamma_{0}$ to $\gamma_{4}$, are

$$
\begin{aligned}
\gamma_{0} & =e^{-\Delta t / \tau} \\
\gamma_{1} & =-\Delta t e^{-\Delta t / \tau}, \\
\gamma_{2} & =1-e^{-\Delta t / \tau} \\
\gamma_{3} & =\Delta t+\tau e^{-\Delta t / \tau}-\tau, \\
\gamma_{4} & =-\tau+\Delta t e^{-\Delta t / \tau}+\tau e^{-\Delta t / \tau} .
\end{aligned}
$$


The flux in the $x_{2}$-direction, $\tilde{\mathbf{F}}_{2}$, can be found similarly as in (20) by changing the coordinate axis and by replacing $c_{1}$ and $\partial / \partial x$ with $c_{2}$ and $\partial / \partial x_{2}$ in the integrand.

For the two-dimensional calculation, we use a dimensional splitting Godunov scheme, [23]. In the scheme, the discretized equations (7) is updated by

$$
\begin{aligned}
\mathbf{W}_{(i, j)}^{n+1}-\mathbf{W}_{(i, j)}^{n}= & \frac{1}{\Delta x_{1}} \int_{t^{n}}^{t^{n+1}}\left(\mathbf{F}_{1\left(i+\frac{1}{2}, j\right)}-\mathbf{F}_{1\left(i-\frac{1}{2}, j\right)}\right) d t+ \\
& \frac{1}{\Delta x_{2}} \int_{t^{n}}^{t^{n+1}}\left(\mathbf{F}_{2\left(i, j+\frac{1}{2}\right)}-\mathbf{F}_{2\left(i, j-\frac{1}{2}\right)}\right) d t+ \\
& \int_{t^{n}}^{t^{n+1}} \Psi_{(i, j)} d t
\end{aligned}
$$

where the superscript ${ }^{n}$ stands for the $n$th time step and the subscripts $i$ and ${ }_{j}$ stand for the grid cells in the $x_{1}-$ and $x_{2}$-directions. A van Leer limiter is applied for the second order reconstruction. The marching time step, $\Delta t$, is constraint by the CFL (Courant-Friedrichs-Lewy) condition with a CFL number 0.5. The relaxation time $\tau$ is calculated following $\mathrm{Xu}[9]$,

$$
\tau=a_{1} \Delta t+a_{2}\left|\frac{h_{l}^{2}-h_{r}^{2}}{h_{l}^{2}+h_{r}^{2}}\right| \Delta t,
$$

where $a_{1}$ and $a_{2}$ are set to be 0.05 and 1.0. Variations of the relaxation time are verified insignificant to the simulation results. After each time step update, the equilibrium particle distribution is disturbed, the numerical scheme resets the initial condition of the particle distribution function and iterate through the aforementioned redistribution process. Then the iteration continues till the terminal time is reached.

Before proceeding further, we recapitulate the derivation of the derivatives of the distribution function for the second order accuracy. These derivatives include $\partial g_{0}^{l, r} / \partial x, \partial \tilde{g}^{l, r} / \partial x$ and $\partial \tilde{g} / \partial t$ in (14) and (15). Because they bear a great deal of similarities, only one essential expression is shown here, $\partial g / \partial x$ for example. Taking $\partial / \partial x$ on (10) and applying the chain rule of calculus, we have

$$
\frac{\partial g}{\partial x}=\left(m_{1}+m_{2} c_{1}+m_{3} c_{2}+m_{4} c_{1} c_{2}+m_{5} c_{1}^{2}+m_{6} c_{2}^{2}\right) g,
$$

where $c_{1}$ and $c_{2}$ are the velocity components of the micro-particles. The coefficients, $m_{1}$ to $m_{6}$, can then be elaborated in terms of the continuum flow variable $\mathbf{W}$ :

$$
\begin{aligned}
m_{1} & =\frac{1}{g_{3} h^{2} k_{1} k_{2}}\left(3\left(k_{22} u_{1}^{2}+2 k_{12} u_{1} u_{2}+k_{11} u_{2}^{2}\right) \frac{\partial h}{\partial x}-2\left(k_{22} u_{1}+k_{12} u_{2}\right) \frac{\partial h u_{1}}{\partial x}-2\left(k_{12} u_{1}+k_{11} u_{2}\right) \frac{\partial h u_{2}}{\partial x}\right), \\
m_{2} & =\frac{1}{g_{3} h^{2} k_{1} k_{2}}\left(-4\left(k_{22} u_{1}+k_{12} u_{2}\right) \frac{\partial h}{\partial x}+2 k_{22} \frac{\partial h u_{1}}{\partial x}+2 k_{12} \frac{\partial h u_{2}}{\partial x}\right), \\
m_{3} & =\frac{1}{g_{3} h^{2} k_{1} k_{2}}\left(-4\left(k_{12} u_{1}+k_{11} u_{2}\right) \frac{\partial h}{\partial x}+2 k_{12} \frac{\partial h u_{1}}{\partial x}+2 k_{11} \frac{\partial h u_{2}}{\partial x}\right), \\
m_{4} & =\frac{1}{g_{3} h^{2} k_{1} k_{2}}\left(2 k_{12} \frac{\partial h}{\partial x}\right), \\
m_{5} & =\frac{1}{g_{3} h^{2} k_{1} k_{2}}\left(k_{22} \frac{\partial h}{\partial x}\right), \\
m_{6} & =\frac{1}{g_{3} h^{2} k_{1} k_{2}}\left(k_{11} \frac{\partial h}{\partial x}\right) .
\end{aligned}
$$

Simply by replacing $\partial / \partial x$ in (24) and (25) with $\partial / \partial t, \partial g / \partial t$ is obtained. For the aforementioned derivatives, the flow variables $\mathbf{W}_{i}\left(x_{i+1 / 2}\right)$ and $\partial \mathbf{W}_{i}\left(x_{i+1 / 2}\right) / \partial x$ are substituted for $\partial g_{0}^{l} / \partial x$ and $\tilde{\mathbf{W}}_{i}\left(x_{i+1 / 2}\right)$, 
$\partial \tilde{\mathbf{W}}_{i}\left(x_{i+1 / 2}\right) / \partial x$ are for $\partial \tilde{g}^{l} / \partial x$ in the $i$ th cell. Similarly, the flow variables in the $(i+1)$ th cell are applied for the derivatives at the immediate right side of the interface and $\tilde{\mathbf{W}}_{i+1 / 2}\left(x_{i+1 / 2}\right), \partial \tilde{\mathbf{W}}_{i+1 / 2}\left(x_{i+1 / 2}\right) / \partial t$ are applied for $\partial \tilde{g} / \partial t$ on the interface. The above abbreviated details conclude the calculation of the derivatives.

\section{Numerical Examples}

Numerical calculations based on the gas kinetic scheme are presented in this section. They are sequentially arranged to illustrate: 1 . verification of the gas kinetic scheme, 2. demonstration of the coordinatedependent Savage-Hutter (SH) equation, 3. the axisymmetric dam break of the anisotropic SH equation and 4. the further influence of the anisotropic SH equation by a sliding mass example. Because the anisotropic properties of the SH model can only be demonstrated using two-dimensional examples and to focus on these properties, the fundamental one-dimensional cases as well as the advantages of the gas kinetic scheme are relegated to Appendix B for the interested readers.

\subsection{Shallow water dam break}

The accuracy of the scheme is first checked using an axisymmetric dam break flow. This example is taken from Chap. 21, page 484 in LeVeque [5] and it is a dam break flow of a cylindrical Eulerian fluid column on a frictioless horizontal basal surface. The fluid column is centered at the origin and the ambient is a resting flow at a uniform depth. The initial condition is

$$
\left(h\left(x_{1}, x_{2}\right), u_{1}\left(x_{1}, x_{2}\right), u_{2}\left(x_{1}, x_{2}\right)\right)= \begin{cases}(1.0,0,0) & r \geq 0.5 \\ (2.0,0,0) & r<0.5\end{cases}
$$

where $r=\left(x_{1}^{2}+x_{2}^{2}\right)^{1 / 2}$. The computational domain is in $x_{1} \in[-2.5,2.5]$ and $x_{2} \in[-2.5,2.5]$. The domain is discretized into a $250 \times 250$ mesh. The out-flow condition is applied on the domain boundaries.

Fig. 2 shows the contour plot of the flow depth and the scatter plots of the radial depth and momentum of the flow. The simulated result is compared to that obtained by 1drad (CLAWPACK, http://www.amath.washington.edu/ ${ }^{\sim}$ claw/. The time instance is $t=1.5$. The minor discrepencies and the smear of the shock wave are because the mesh in the gas kinetic scheme is much coarser than that used in $1 \mathrm{drad}$, (250 compared to 2000). It is confirmed that the simulation converges to $1 \mathrm{drad}$ when the number of grids is increased. The scatter plots show that the axisymmetry of the flow is preserved.

\subsection{Coordinate dependence of the Savage-Hutter model}

The second example is to demonstrate the intrinsic coordinate dependence of the Savage-Hutter model. In the model, it is assumed that the primary earth pressure coefficient $\left(k_{1}\right)$ is applied in the main direction of the flow, say $x_{1}$-direction, and the secondary earth pressure coefficient $\left(k_{2}\right)$ is in the transverse $\left(x_{2}-\right)$ direction. The off-diagonal earth pressure coefficients are neglected. While this approach achieved substantial successes for avalanche flows, [18], [24], the role of the neglected off-diagonal shearing components remains largely unnoticed. The negligence of the shearing components leads to a coordinate dependent property of the model. This property can be seen clearly in a collapsing flow on a horizontal plane because no preferred flow direction is present.

To illustrate this coordinate dependent property, a collapsing flow of an initially axisymmetric top-hat shaped fluid colume is simulated. The radius and height are set to 2.0 and 3.0 and the external ambient is a dry field. The initial condition reads

$$
\left(h, u_{1}, u_{2}\right)= \begin{cases}(0,0,0) & r \geq 2.0 \\ (3,0,0) & r<2.0\end{cases}
$$


(a)

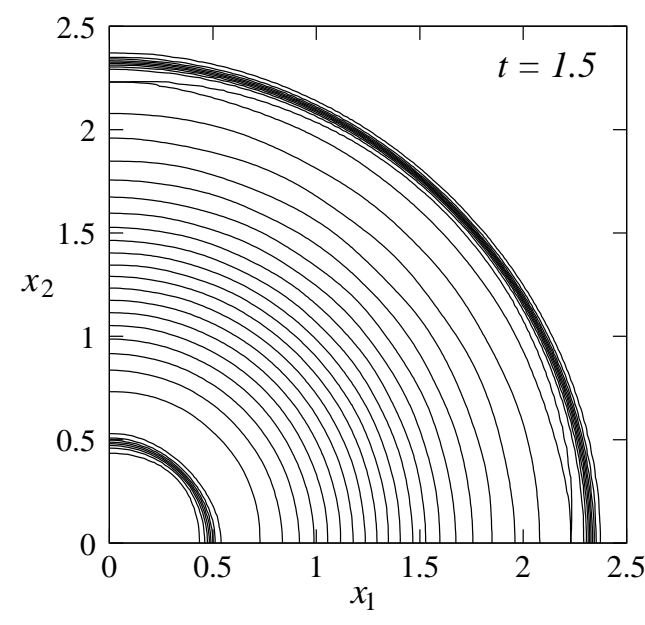

(b)

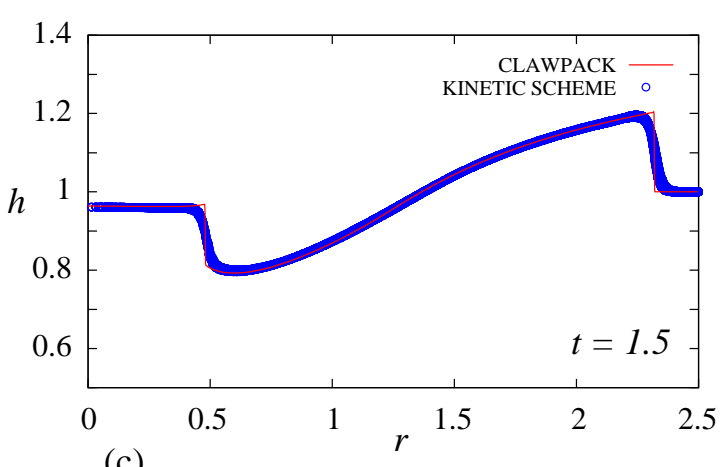

(c)

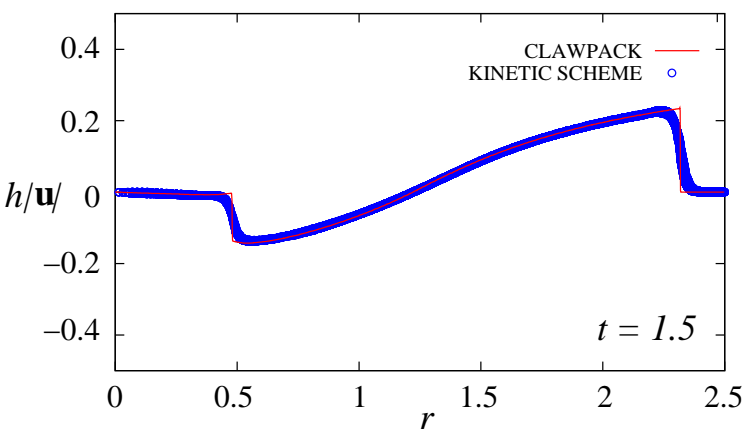

Figure 2: The snapshot of the axisymmetric dam break flow at $t=1.5$. (a), The flow depth contour. Contour levels are from 0.61 to 1.31 at a spacing 0.02 . (b) The radial scatter plot of the flow depth. (c) The radial scatter plot of the radial momentum. The red solid line in the scatter plot is the $1 \mathrm{D}$ solution of CLAWPACK and the blue circle points are the $2 \mathrm{D}$ result of the present scheme.

The basal surface is a horizontal flat plane. Because this flow is an expanding flow and no shock waves are formed, the active earth pressure coefficients, i.e. taking the minus signs in (5) and (6), are chosen. The internal friction angle $\phi$ and the basal friction angle $\delta$ are both set to $30^{\circ}$ in the simulation. These values lead to the two earth pressure coefficients $k_{1}=1.67$ and $k_{2}=0.45$. The computational domain is $x_{1} \in[-20,20]$ and $x_{2} \in[-20,20]$ and it is discretized into 400 cells in each direction.

The numerical result is sketched in Fig. 3 and the axes are set to $[-5,5]$ for clarity. The simulation of the shallow water equation on the $x_{1}$ and $x_{2}$ profiles have also been plotted for comparison. The striking finding is that the fluid column turns into an oval shape and the primary axis coincides with the $x_{1}$ axis. The cause of the shape is because of the allignment of the earth pressure coefficients and the coordinates. In this example, the principal earth pressure coefficient is alligned in the $x_{1}$-direction and the secondary is in the $x_{2}$-direction. This difference between the principal and secondary earth pressure coefficients leads to different wave speeds in the coordinate directions. Because the wave speeds are roughly proportional to $\sqrt{k_{1} g_{3} h}$ and $\sqrt{k_{2} g_{3} h}$ along the two coordinates, the ratio between the wave speeds is estimated about 1.93. The larger wave speed occurs in the $x_{1}$-direction and it subsequently affects the aspect ratio of the flow depth outline. The orientation of the oval shape depends on the direction in which $k_{1}$ is chosen and the Savage-Hutter model is, therefore, coordinate dependent.

This coordinate dependence leads to a non-objective property of the Savage-Hutter model. It is an important intrinsic property of the model which manifests itself when the flow has no preferred direction of motion. This coordinate-dependent non-objective property can be resolved by including the tangential shear stresses, i.e. via the off-diagonal earth pressure coefficients. A few theoretical alternatives have been proposed for this purpose in literature. For example, De Toni and Scotton [15] assume that the 
(b)

(a)

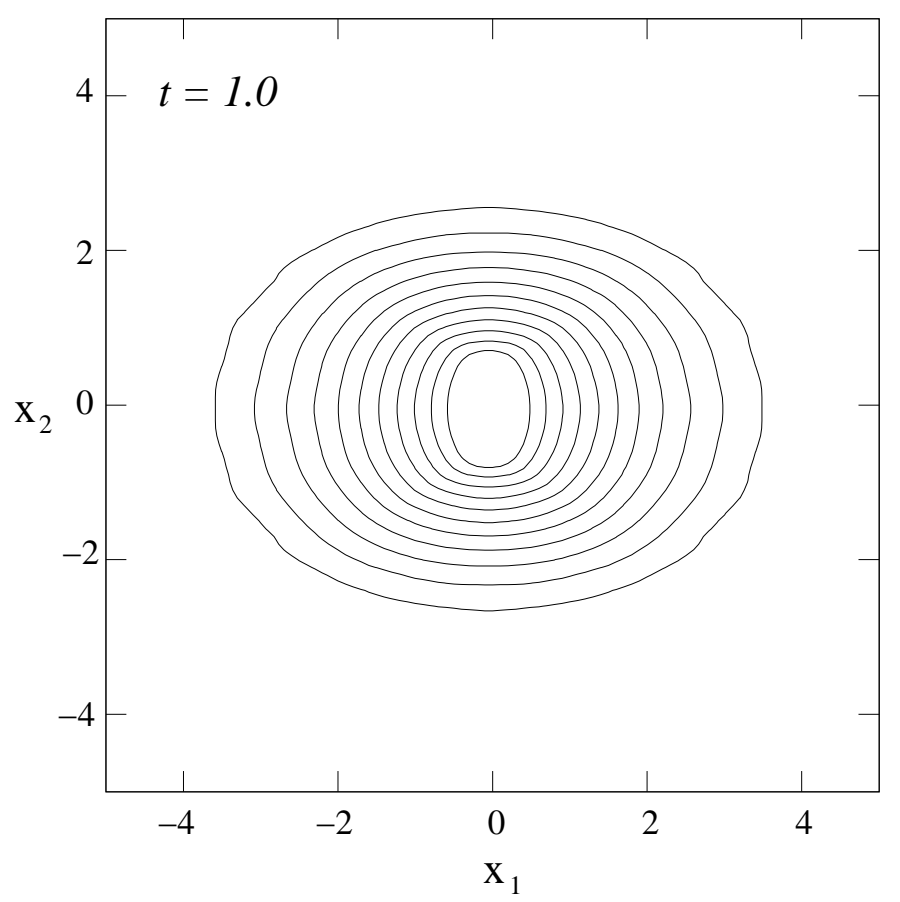

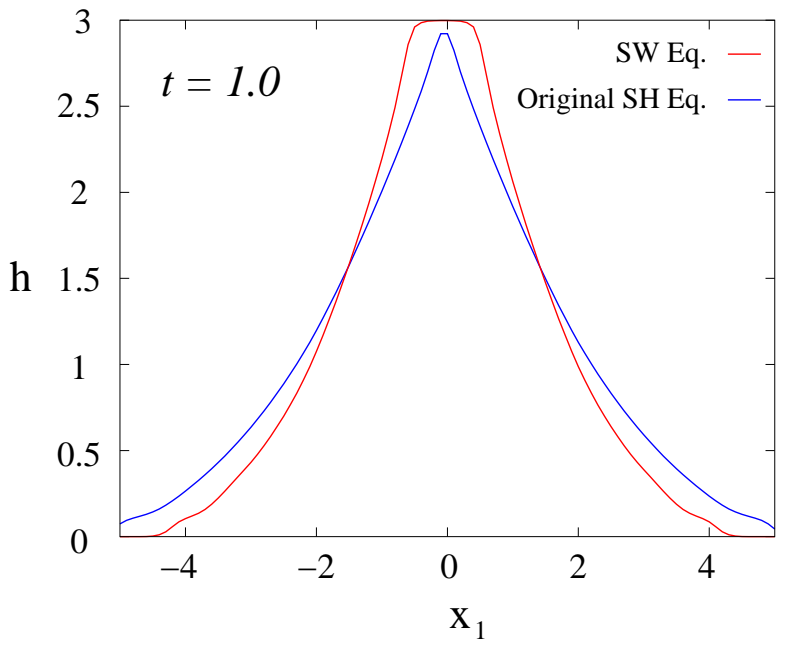

(c)

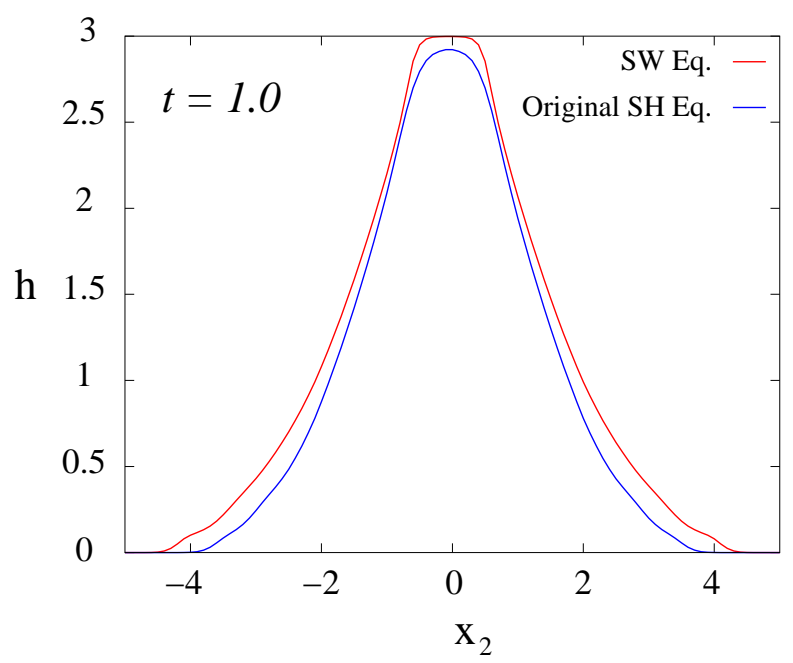

Figure 3: Coordinate dependent Savage-Hutter model. The flow snapshot is taken at $t=1$. The primary principal axis is $x_{1}$. (a) Contour of flow depth. The contour level monotonically decreases from a depth value 2.4 (innermost) at a level step -0.2. Comparison of the Savage-Hutter (SH) model and the shallow-water (SW) equation on (b) $x_{1}$-axis and (c) $x_{2}$-axis.

primary principal axis is in parallel to the local flow velocity and Luca et al. [12] define that the principal axes coincides with those of the local strain rate. In the present paper, the former approach is adopted without loss of generality and two example will be presented to illustrate the effects of the anisotropic stresses.

\subsection{Axisymmetric dam break flow}

In this section, an axisymmetric dam break of the Savage-Hutter model is investigated. Because of the axial symmetry, the primary principal axis is in the radial direction. For the discretized numerical simulation, it is found that the orientation of the principal axes in the adjacent computation cells surrounding the axisymmetric center varies dramatically and it produces a mild numerical singularity at the center. Without being digressed from the singularity in the present paper, an inward axisymmetric dam break 
(a)

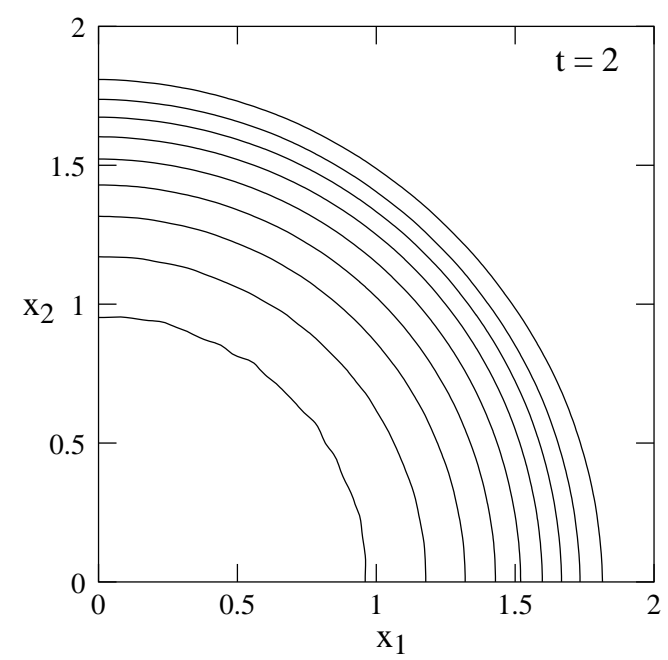

(b)

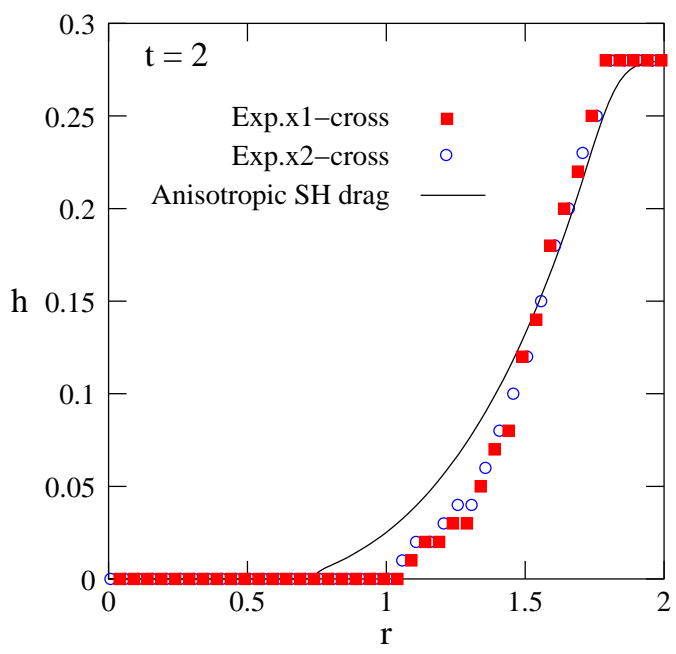

Figure 4: Axisymmetric inward dam break flow. The flow snap is taken at $t=2.0$. (a) Contour of flow depth. The axisymmetry is resumed by binging in the effect of the principal axis orientation. Contour levels are from 0.02 (innermost) to 0.26 at a level step 0.03 .

is simulated instead. The initial condition (dimensionless) is defined as

$$
\left(h, u_{1}, u_{2}\right)= \begin{cases}(0.27,0,0) & r \geq 1.5 \\ (0,0,0) & r<1.5\end{cases}
$$

The basal surface is a horizontal flat plane. The basal and the internal friction angles are $28^{\circ}$ and $34^{\circ}$, which yield the earth pressure coefficients $k_{1}^{a c t}=0.91$ in the radial direction and $k_{2}^{a c t}=0.42$ in the circumferential direction. The computational domain is $x \in[-2,2]$ and $y \in[-2,2]$ and is discretized into 200 cells in each direction.

Because the orientation of the primary principal axis is in the radial direction, the rotation matrix $\mathbf{T}(\theta)$ defined in obtaining (10) is determined accordingly

$$
\mathbf{T}(\theta)=\left(\begin{array}{cc}
\cos \theta & -\sin \theta \\
\sin \theta & \cos \theta
\end{array}\right)
$$

where $\theta$ here is the angular coordinate of the position vector. In this example, the simulation continues till the flow ends. To ensure the terminal resting deposit, a numerical stationary condition, similar to Chap. 4 [25], is used. The minor difference is that the friction force is limited by the rate of momentum in the present scheme instead of by the rate of energy.

Figure 4 shows the numerical result of the deposit. The contour of the deposit depth indicates that the axisymmetric shape is retained. In Fig. 4(b), the simulation is compared to the experimental measurement. The simulation spreads wider than the experiment. This discrepency can be expected because in Savage-Hutter model the flow is assumed to have a uniform velocity profile while in the experiments a full three-dimensional flow develops, especially around the edge of the initial flow. In summary, this example demonstrates that the coordinate objectivity is preserved by properly including the orientation of the principal axes of the anisotropic tangential stresses.

\subsection{Finite mass sliding down an inclined planary chute}

In the last example, the sliding of a finite mass of granular material is calculated. The mass has an initially resting semi-spherical shape. The base radius is $r_{0}=1.85$ (dimensionless), centered at $x_{1}=4$ 


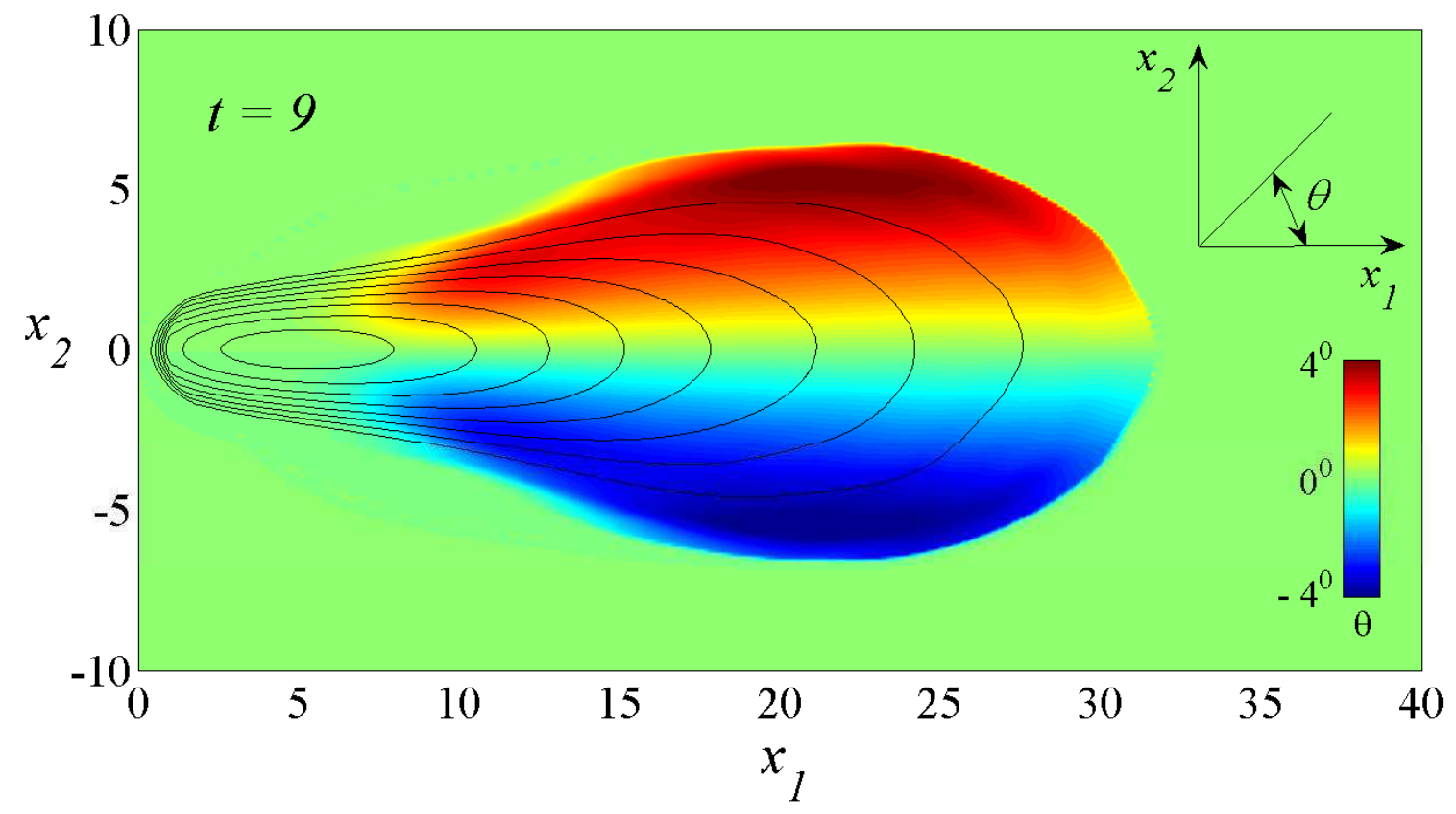

Figure 5: Orientation of the primary principal axis of the anisotropic Savage-Hutter model. The flow is a finite mass sliding down an inclined planary chute and the flow snap is taken at $t=9$. The orientation of the principal axis is defined by the angle from $x_{1}$. Contour lines are for the flow depth with levels from 0.02 to 0.16 at 0.02 .

and $x_{2}=0$. At $t=0$, the mass is released and slides down a planary chute, inclined at $35^{\circ}$. The initial condition of the initial shape is

$$
\left(h, u_{1}, u_{2}\right)= \begin{cases}(0,0,0) & r \geq r_{0} \\ \left(\left(r_{0}^{2}-r^{2}\right)^{1 / 2}, 0,0\right) & r<r_{0} .\end{cases}
$$

Both of the internal and the basal friction angle are $30^{\circ}$, which yields $k_{1}=1.67$ and $k_{2}=0.45$. The initial primary principal axis is assumed in parallel with the inclination direction, the $x_{1}$-axis. The computational domain is set $x \in[0,60]$ and $x_{2} \in[-20,20]$ divided into 600 and 400 cells. This domain is set sufficiently large to observe the development of the principal axes of the stresses of the sliding flow.

Shortly after the mass being released, the flow starts spreading laterally and the primary principal axis shifts accordingly. The orientation of the primary principal axis and the flow contour at $t=9.0$ is sketched in Fig. 5. In the figure, the orientation of principal axis is symmetric with respect to the central line at a maximum deflected angle about $\pm 4^{\circ}$ near the widest flanges of the flow. Mass conservation is also checked. The mass loss from the start of simulation is plotted in Fig. 6. Before the sliding material begins to leave the computation domain, it is seen that the mass loss is well confined within $10^{-8}$ (actually $10^{-10}$, relative to the total mass), i.e. within the margin of the numerical round-off error.

Finally, the simulation of the anisotropic Savage-Hutter (SH) model is compared with those of the original (coordinate-dependent) SH equation and the shallow water equation. The outlines and flow profiles of the sliding flow at the same time instance, $t=9.0$, as in Fig. 5 are plotted in Fig. 7 . With the original SH equation, the primary earth pressure coefficient $k_{1}$ is assumed in the $x_{1}$-direction and its large value (1.67) leads to the faster traveling speed of the flow front than the other models in the down chute direction. In addition to the small secondary earth pressure coefficient $k_{2}$ in the $x_{2}$-direction, the original SH equation yields the most slender flow outline at the time instance. 


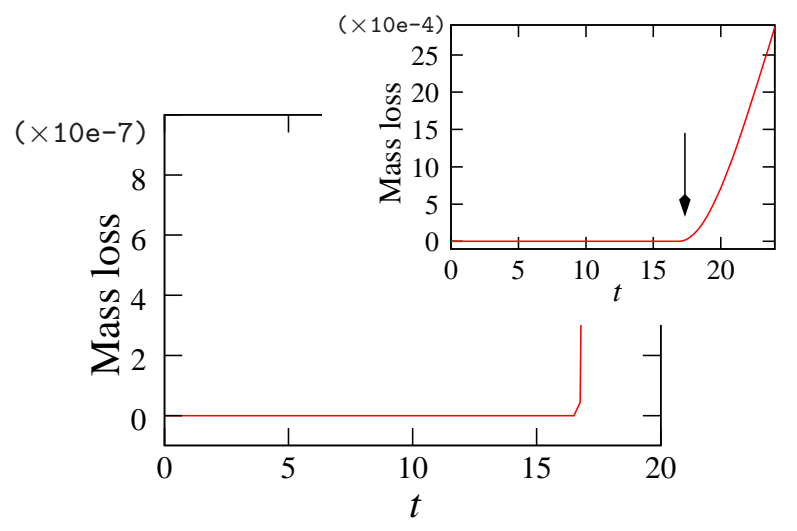

Figure 6: The mass conservation of the sliding mass. The line shows the mass loss from the start of the simulation. The vertical solid arrow in the inset figure indicates the instance that the flow starts to leave the computation domain.

If the earth pressure coefficient matrix is set to an identy matrix, the Savage-Hutter equation degenerates into the isotropic shallow water equation. In this case, the flow spreads more uniformly in every direction as seen from the more roundish outline. The anisotropic SH model, on the other hand, lies between the other two alternatives. The reason is plausible now because the change of the orientation of the stress principal axes towards the flow velocity in the anisotropic SH model leads to the higher lateral spreading speed and the lower flow front speed than the original SH equation. The depth profiles in Fig. 7(b,c) indicate that the shallow water equation has the highest overall spreading rate (measured by the spreading area divided by the maximum flow height).

\section{Conclusion}

In this paper, the gas-kinetic scheme is applied to the general anisotropic Savage-Hutter equation for modeling the avalanche flows. The solid properties of the landslide materials are modeled by the anisotropic tangential stress which is related to the flow depth via the matrix of the earth pressure coefficients. With the anisotropic stress and under the maximum entropy condition, the equilibrium distribution of the pseudo particles is found a bi-Maxwellian function. Assuming that the distribution of the pseudo particles satisfies the Bhatnagar-Gross-Krook equation, the continuum Savage-Hutter equation can be obtained by carrying out the kinetic moment integration over the particle motions and hence a discretized numerical scheme is developed.

The coordinate dependence of the original Savage-Hutter model is first demonstrated. This nonobjectivity results from the neglect of the tangential shear stress. By incorporating the principal stress states, i.e. the effect of the shear stress, the deposit of the inward axisymmetric dam break flow resumes axisymmetric, and, hence, the coordinate depdence property is removed. Finally, the simulation of a finite mass sliding down an inclined planary chute is performed. The results of the anisotropic SavageHutter model, the original SH equation and the shallow water equation are compared. It is found that the spreading of the sliding flow significantly depends on the effect of the stress states. The benefits of this scheme: the capability to resolve the shock discontinuities sharply and to handle the vacuum state, are also successfully retained for the anisotropic Savage-Hutter model. 
(b)

(a)

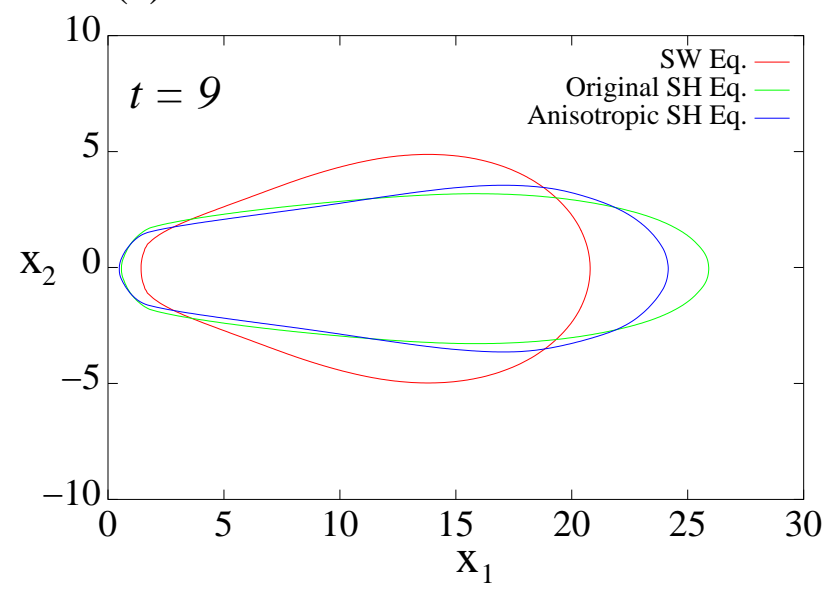

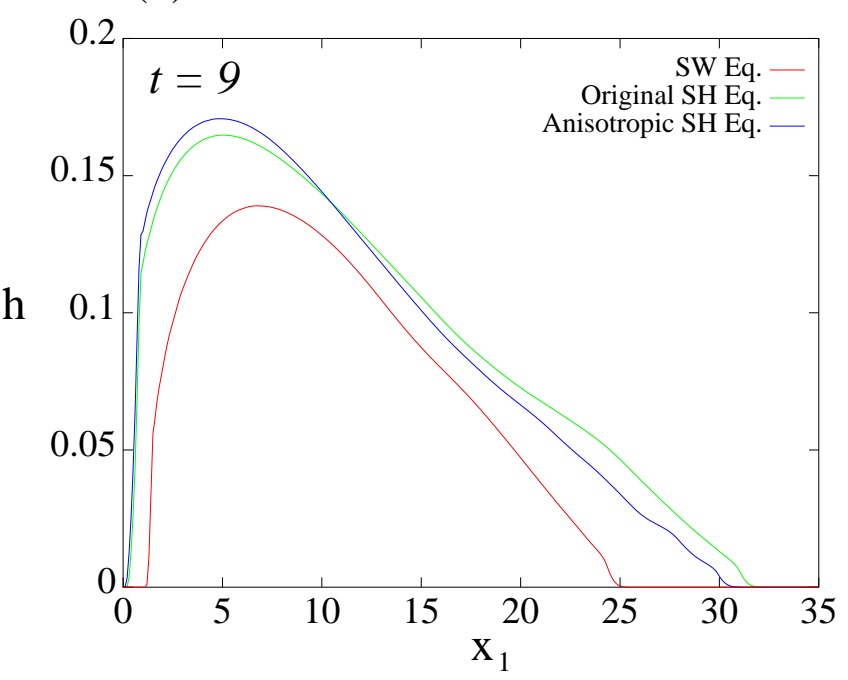

(c)

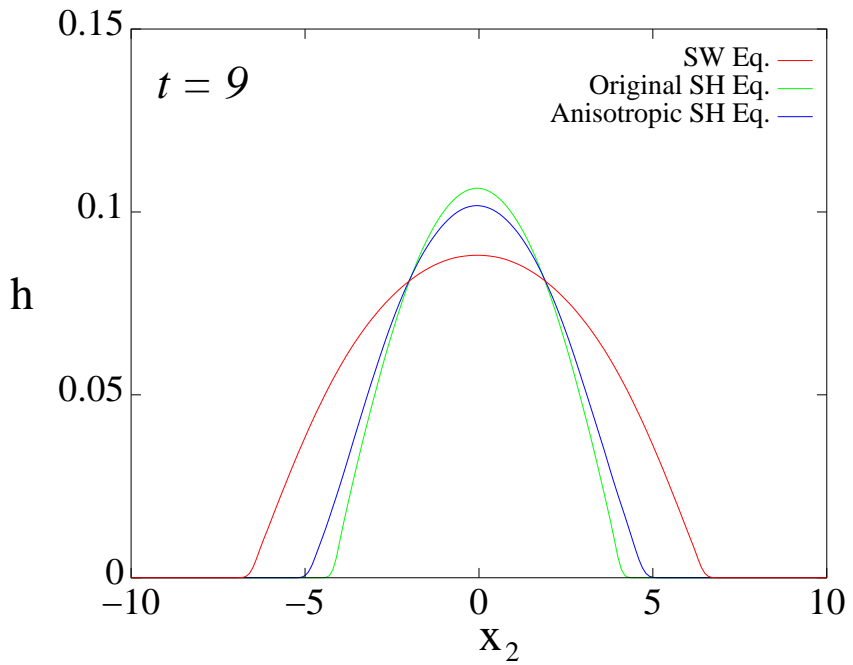

Figure 7: A finite mass sliding down an inclined planary chute. The inclination angle is $35^{\circ}$. Comparison is made among the anisotropic Savage-Hutter ( $\mathrm{SH}$ ) model, the original coordinate-dependent $\mathrm{SH}$ equation and the shallow water (SW) equation. (a) Flow outlines, (b) depth profile on the $x_{1}$-axis and (c) transverse depth profile at $x_{1}=15$.

\section{Acknowledgements}

This work is supported in part by National Science Council, Taiwan (NSC 99-2116-M-001-015-, 100-2116M-001-008-).

\section{A Bi-Maxwellian distribution}

We assume that the process of a collection of pseudo-particles from an non-equilibrium state to the equilibrium state follows the BGK equation, (8), where $f$ and $g$ correspond to the non-equilibrium and equilibrium distributions of the pseudo-particles, respectively. When the particles reach equilibrium, we have $f=g$ and $D f / D t=0$, i.e. the collision term on the righ hand side of (8) vanishes. 
The continuum physical quantities are the collective resultants of the pseudo-particles, which include the flow depth, $h$, momentum, $h \mathbf{u}$, momentum flux, $h\left(\mathbf{u} \otimes \mathbf{u}-g_{3} \mathbf{K} h / 2\right)$, and the entropy $S$, and they are

$$
\begin{aligned}
h & =\int f d \mathbf{c}, \\
h \mathbf{u} & =\int \mathbf{c} f d \mathbf{c}, \\
h \mathbf{u} \otimes \mathbf{u}-\frac{1}{2} g_{3} \mathbf{K} h^{2} & =\int \mathbf{c} \otimes \mathbf{c} f d \mathbf{c}, \\
S & =-\int f \ln (A f) d \mathbf{c},
\end{aligned}
$$

where $A$ is a normalizing constant. Note that matrix $\mathbf{K}$ is a $2 \times 2$ diagonal matrix, as defined in Sec. 2 and its diagonal elements are the earth pressure coefficients $k_{1}$ and $k_{2}$ defined in (5), (6) in the Savage-Hutter model. According to the second law of thermodynamics, the entropy $S$ monotonically increases with time and reaches the maximum value when at equlibrium. This is to say

$$
\frac{D S}{D t} \geq 0, \text { for all } f, \text { and } \delta S=0 \text { when } f=g,
$$

where $\delta S$ is the variation of the entropy. The maximization of $S$, subjected to constraints from (26) to (28), is performed with the aid of Lagrange multipliers. This yields

$$
\begin{aligned}
S= & -\int\left(f \ln (A f)+\lambda_{0} f+\boldsymbol{\lambda} \cdot \mathbf{c} f+f(\mathbf{c}-\mathbf{u})^{T} \boldsymbol{\Gamma}(\mathbf{c}-\mathbf{u})\right) d \mathbf{c} \\
& +\lambda_{0} h+\boldsymbol{\lambda} \cdot \mathbf{u} h-\frac{g_{3} h^{2}}{2} \boldsymbol{\Gamma}: \mathbf{K}
\end{aligned}
$$

where $\lambda_{0}, \boldsymbol{\lambda}$ and $\boldsymbol{\Gamma}$ are the scalar, vector, and tensorial multipliers. Following the same procedures as in [26], we obtain the bi-Maxwellian equilibrium distribution

$$
g=\frac{1}{A} \exp \left(-1-\lambda_{0}\right) \exp \left[-(\mathbf{c}-\mathbf{u})^{T} \boldsymbol{\Gamma}(\mathbf{c}-\mathbf{u})\right]
$$

where the Lagrange multipliers and the normalizing constant are

$$
\begin{aligned}
\lambda_{0} & =-2, \\
\boldsymbol{\lambda} & =\mathbf{0} \\
\boldsymbol{\Gamma} & =\frac{1}{g h} \mathbf{K}^{-1} \\
A & =\pi g_{3} \sqrt{k_{1} k_{2}} \exp (1) .
\end{aligned}
$$

\section{B One-Dimensional Numerical Tests}

We verify the kinetic scheme and demonstrate the effect of the earth pressure coefficient for onedimensional flows in this section.

\section{B.1 Inviscid Dam break Flows}

The shallow water equation on a horizontal flat bed is solved. The water flow is inviscid and frictionless on the basal surface. Two test problems are calculated and their initial conditions are, respectively,

$$
(h, u)= \begin{cases}(3,0) & x \leq 0 \\ (0,0) & r>0,\end{cases}
$$


and

$$
(h, u)= \begin{cases}(3,0) & x \leq 0 \\ (1,0) & r>0 .\end{cases}
$$

The computational domain is set between $-5 \leq x \leq 5$ and discretized into 500 grid points. The constant depth condition and the outflow condition are applied to the upstream and downstream ends of the calculation domain. The solution of $h$ and $h u$ at $t=1.0$ is shown in Fig. 8 and Fig. 9, where the solid lines are the analytical solutions and the circles are the numerical simulation.

The first example corresponds to a dam break flow into a dry (vacuum) domain. It is confirmed that the solution of the gas-kinetic scheme converges to the analytical solution and the scheme handles the dry state intrinsically without the need of any special modifications.

The second example is similar to the classical shock tube problem in the gas dynamics. After the dam break, the flow generates two waves: one donwstream propagating shock wave, as the weak solution of the SW equations, and one upstream propagating expansion wave, as shown in Fig. 9. The discontinous shock is also resolved accurately with the scheme of the second order accuracy. These two examples concludes the advantages of the gas kinetic scheme: vacuum handling and shock capturing. In addition, the positiveness of the flow depth is theoretically ensured, Tang et al. [27].

(a)

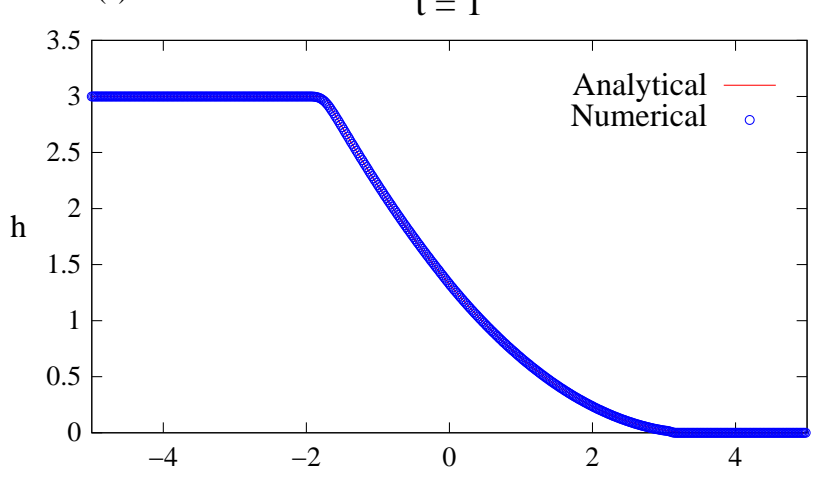

(b)

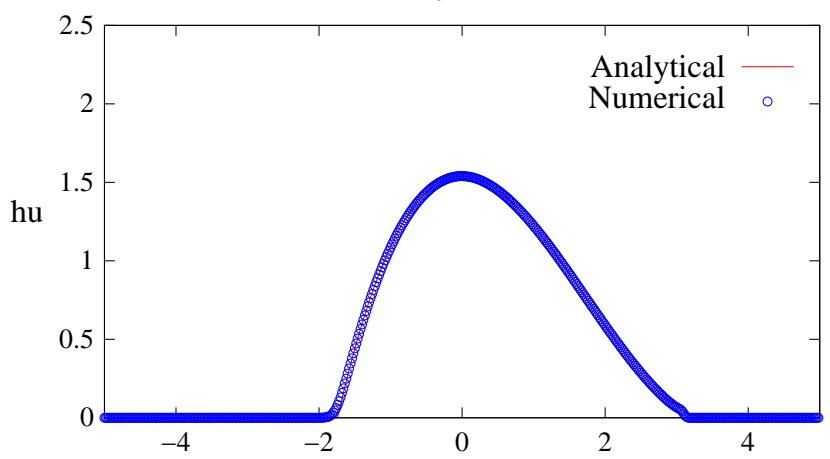

Figure 8: The analytical and numerical solutions of (a) the flow depth $h$, and (b) the momentum $h u$ at $t=1.0$ of the SW model on wet-dry bed, (32). The computation domain is discretized into 500 cells. The basal surface is a horizontal flat plane.
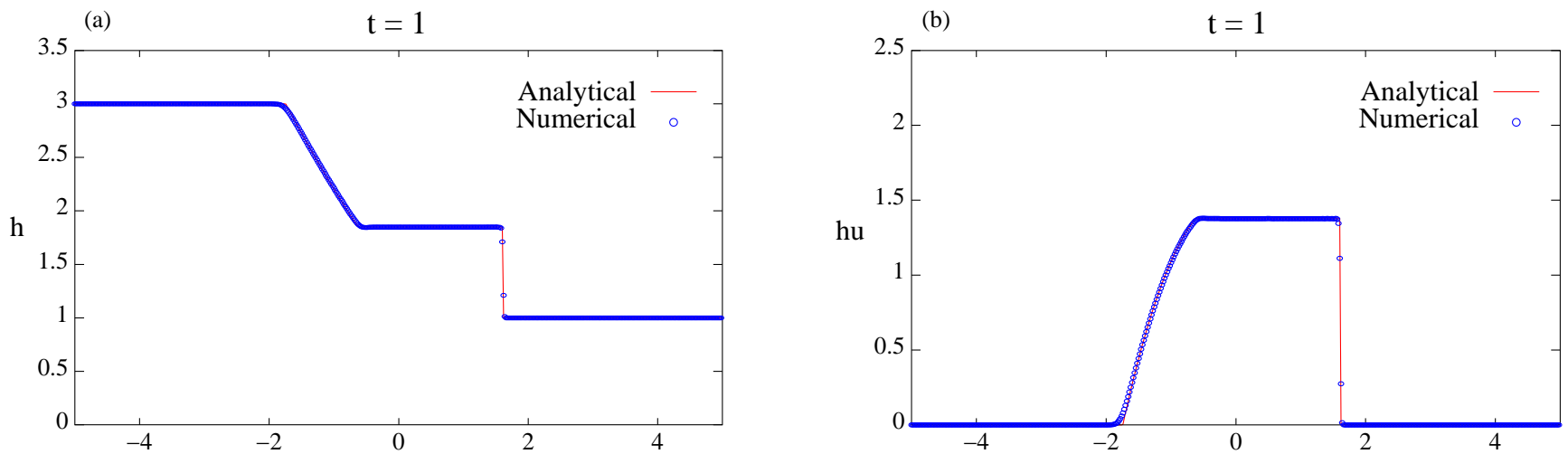

Figure 9: The analytical and numerical solutions of (a) the flow depth $h$, and (b) the momentum $h u$ at $t=1.0$ of the SW model on wet-wet bed, (33). The computation domain is discretized into 500 cells. The basal surface is a horizontal flat plane. 
(a)

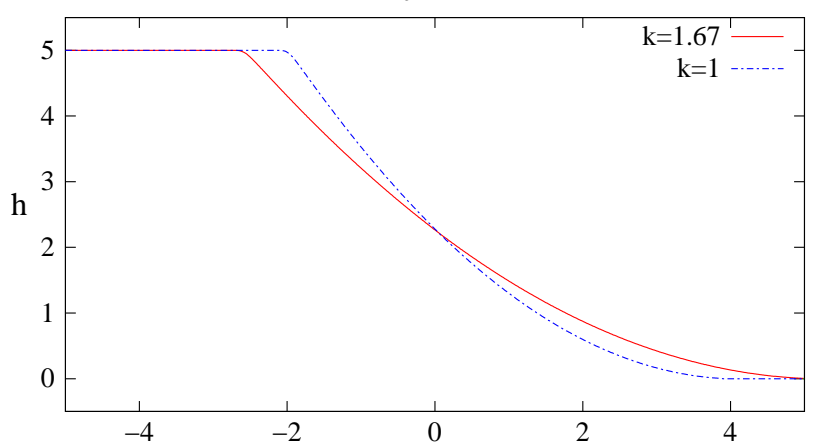

(b)

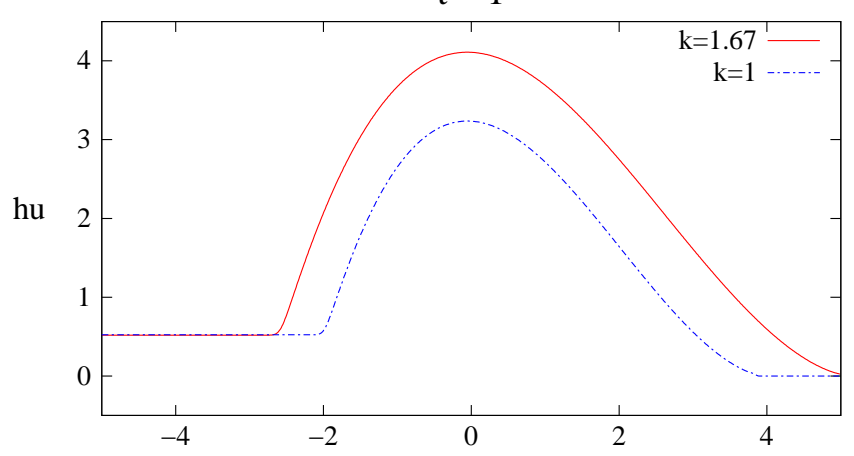

Figure 10: Influence of earth pressure coefficient. The coefficients are $k=1.0$ and $k=1.67$, corresponding to the shallow water and Savage-Hutter models, respectively. (a) Flow depth $h$, (b) momentum $h u$ at $t=1.0$ on a wet-dry bed, (34). The computation domain is discretized into 1000 cells. The basal surface is inclined at $\zeta=35^{\circ}$ and the basal and internal friction angles are $\delta=\phi=30^{\circ}$.

\section{B.2 Effect of Earth Pressure Coefficient}

We now simulate the dam break flows of the Savage-Hutter model and compare with the shallow water model. The main purpose is to illustrate the effect of the earth pressure coefficient. In the one-dimensional flow, only the primary coefficient, (5), is needed. Two test examples are calculated and they are arranged similarly to those in Sec. B.1: an expansion flow into the dry state and a shock wave dam break. Both of the internal and basal friction angles, $\phi$ and $\delta$, are set to $30^{\circ}$, which yields $k_{1}^{\text {act }}=k_{1}^{\text {pass }} \approx 1.67$. The flat basal surface is tilted at an inclination angle $\zeta=35^{\circ}$ to let the gravity drive the flow. The initial flow profiles of the two cases are given as follows

$$
(h, u)= \begin{cases}(5,0) & x \leq 0 \\ (0,0) & r>0\end{cases}
$$

and

$$
(h, u)= \begin{cases}(5,0) & x \leq 0 \\ (1,0) & r>0 .\end{cases}
$$

In the correspondent shallow water model, the same basal friction force is applied, such that the only difference between the two models is the earth pressure coefficient. The computational domain is set the same as in Sec. B.1 but is discretized into 1000 grid points. The constant depth condition and the outflow condition are applied to the upstream and downstream ends of the calculation domain.

The flows at $t=1.0$ are shown in Figs. 10 and 11. These results exhibit similar behaviors as those in the previous section. For comparison, the solutions of the shallow water model is plotted as the blue dashed lines. It is clear seen from the position of the wave fronts that both of the shock and expansion wave speeds are higher in the Savage-Hutter model than those in the shallow water model. This is because the large earth pressure coefficient, 1.67, leads to a higher wave speed compare to the shallow water equation. The wave speed of a 1D surface wave of the Savage-Hutter equation can be found $\sqrt{g_{3} k_{1} h}$ following the same procedures as in the shallow-water equations. Accordingly, the spans of the expansion waves are wider than the shallow water counterpart which leads to the milder surface slope. Interestingly, in the intermediate zone between the shock and the expansion wave, Fig. 11, the flow depth does not change. 
(a)

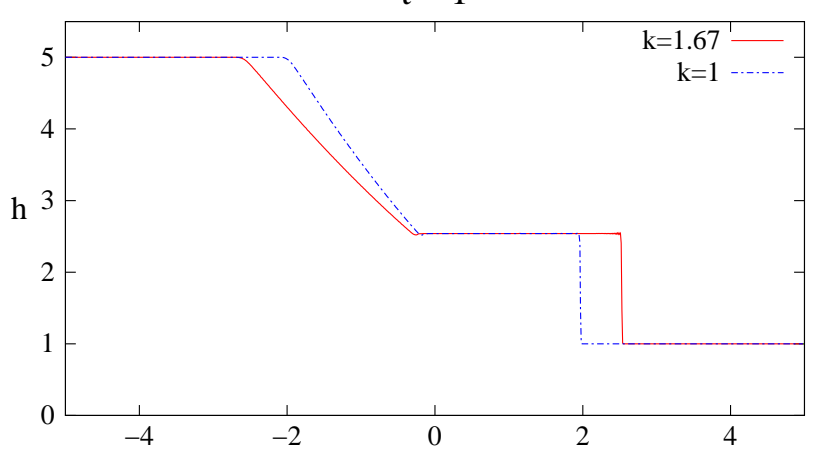

(b)

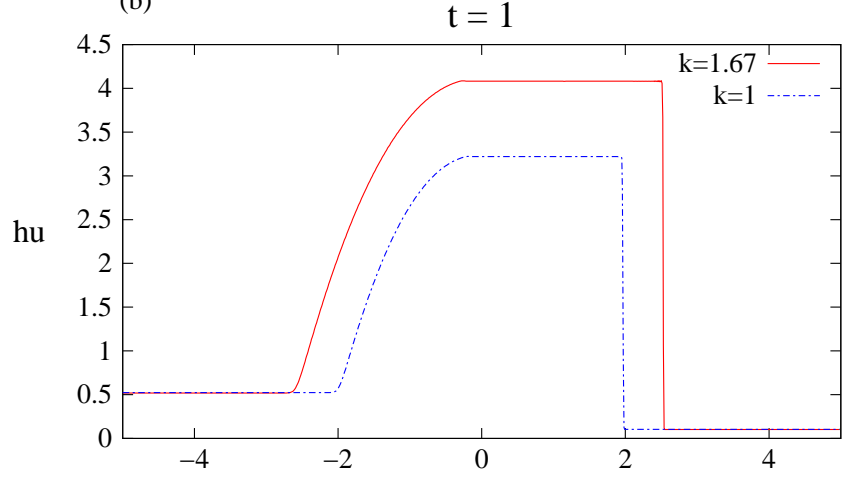

Figure 11: Influence of earth pressure coefficients. The coefficients are $k=1.0$ and $k=1.67$, corresponding to the shallow water and Savage-Hutter models, respectively. (a) Flow depth $h$, (b) momentum $h u$ at $t=1.0$ on a wet-wet bed, (35). The computation domain is discretized into 1000 cells. The basal surface is inclined at $\zeta=35^{\circ}$ and the basal and internal friction angles are $\delta=\phi=30^{\circ}$.

\section{References}

[1] Savage, S. B. and Hutter, K., "The motion of a finite mass of granular material down a rough incline," J. Fluid Mech., Vol. 199, 177-215, 1989.

[2] Hutter, K., Siegel, M., Savage, S. B. and Nohguchi, Y., "Two-dimensional spreading of a granular avalanche down an inclined plane. part i: Theory." Acta Mech., Vol. 100, 37-68, 1993.

[3] Pudasaini, S. P. and Hutter, K., "Rapid shear flows of dru granular massis down curved and twisted channels." J. Fluid Mech., Vol. 495, 193-208, 2003.

[4] Wang, Y., Hutter, K. and Pudasaini, S. P., "The Savage-Hutter theory: A system of partial differential equations for avalanche flows of snow, debris and mud," J. App. Math. Mech., Vol. 84, 507-527, 2004.

[5] LeVeque, R. J., Finite Volume Methods for Hyperbolic Problems, Cambridge Univ. Press, 2003.

[6] Toro, E. L., Riemann Solvers and Numerical Methods for Fluid Dynamics, Springer-Verlag, New York, 1997.

[7] Jiang, G. and Tadmor, E., "Non-oscillatory central schemes for multidimensional hyperbolic conservation laws," SIAM J. Sci. Comput., Vol. 19, 1892-1917, 1997.

[8] Tai, Y. C., Noelle, S., Gray, J. M. N. T. and Hutter, K., "Shock-capturing and front tracking methods for granular avalanches," J. Comput. Phys., Vol. 175, 269-301, 2002.

[9] Xu, K., "A well-balanced gas-kinetic scheme for the shallow-water equations with source terms," $J$. Comp. Phys., Vol. 178, 533-562, 2002.

[10] Gray, J. M. N. T., Wieland, M. and Hutter, K., "Gravity-driven free surface flow of granular avalanches over complex basal topography." Proc. R. Soc. A, Vol. A455, 1841-1874, 1999.

[11] Wieland, M., Gray, J. M. N. T. and Hutter, K., "Channelized free-surface flow of cohesionless granular avalanches in a chute with shallow lateral curvature," J. Fluid Mech., 73-100, 1999. 
[12] Luca, I., Hutter, K., Tai, Y. C. and Kuo, C. Y., "A hierarchy of avalanche models on arbitrary topography," Acta Mech., Vol. 205, 121-149, 2009.

[13] Hutter, K., Wang, Y. and Pudasaini, S., "The Savage-Hutter avalanche model: how far can it be pushed?" Phil. Trans. R. Soc. A, Vol. 363, 1507-1528, 2005.

[14] Iverson, R. M. and Denlinger, R. P., "Flow of variably fluidized granular masses across threedimensional terrain. 1. coulomb mixture theory," J. Geophys. Res., Vol. 106, 537-552, 2001.

[15] De Toni, S. and Scotton, P., "Two-dimensional mathematical and numerical model for the dynamics of granular avalanches," Cold Reg. Sci. Tech., Vol. 43, 36-48, 2005.

[16] Kelfoun, K. and Druitt, T. H., "Numerical modeling of the emplacement of Socompa rock avalanche, Chile," J. Geophys. Res., Vol. 110, B12202, 2005.

[17] Pudasaini, S. P. and Hutter, K., Avalanche dynamics, Springer Verlag, Berlin/Heidelberg, 2007.

[18] Hutter, K., Wang, Y. and Pudasaini, S. P., "The savage-hutter avalanche model: how far can it be pushed," Phil. Trans. R. Soc. A, Vol. 363, 1507-1528, 2005.

[19] Bhatnagar, P. L., Gross, E. P. and Krook, M., "A model for collision processes in gases i: Small amplitude processes in charged and neutral one-component systems," Phys. Rev., Vol. 94, 1954.

[20] Prendergast, K. H. and Xu, K., "Numerical hydrodynamics from gas-kinetic theory," J. Comp. Phys., Vol. 109, 53-66, 1993.

[21] Xu, K., "Gas-kinetic scheme for unsteady compressible flow simulations," Lecture series, von Karman Institute for Fluid Dynamics, Caltech., 1998.

[22] Xu, K. and Guo, Z., "Generalized gas dynamic equations," 47th AIAA Aerospace Sciences Meeting, AIAA 2009-672, 2009.

[23] $\mathrm{Xu}, \mathrm{K} .$, "A gas-kinetic bgk scheme for the navierstokes equations and its connection with artificial dissipation and godunov method," J. Comp. Phys., Vol. 171, 289335, 2001.

[24] Pudasaini, S. P. and Hutter, K., Avalanche Dynamics, dynamics of rapid flows of dense granular avalanches, Springer, 2007.

[25] Bouchut, F., Nonlinear stability of finite volume methods for hyperbolic conservation laws and wellbalanced schemes for sources, Birkhauser Verlag, 2004.

[26] Ghidaoui, M. S., Deng, J. Q., Gray, W. G. and Xu, K., "A Boltzmann based model for open channel flows," Int. J. Numer. Methods Fluids, Vol. 35, 449-494, 2001.

[27] Tang, H., Tang, T. and Xu, K., "A gas-kinetic scheme for shallow-water equations with source terms," ZAMP, Vol. 55, 365382, 2004. 